\section{Overcoming a difficult nasogastric tube insertion procedure with a video laryngoscope (C-Mac ${ }^{\circledR}$ )}

Sir,

Nasogastric tube (NGT) placement is a basic procedure in a critical care unit, but it can be challenging even for experienced anesthesiologists. The failure rate in the first attempt with the blind method has been reported to be as high as $50 \%{ }^{[1]}$ Variations in a patient's functional anatomy, anesthetized, and paralyzed patients, and the presence of endotracheal tube can further complicate an already difficult procedure. Although various techniques have been suggested to make NGT insertion easier, failure to insert or NGT malposition still occurs. The most frequent malpositioning of NGT occurs in the respiratory tract. $^{[2]}$ The complications of an endobronchial misplaced NGT can be very serious, with airway leakage and pulmonary edema, and may even lead to death. A study reported NGT placement in the left or right bronchus in 50 out of 2000 cases over a period of 4 years, with two mortalities. ${ }^{[3]}$

A 50-year-old man, with no known medical illness, was admitted to our intensive care unit for ventilator support due to traumatic brain injury. The patient had an oral cuffed endotracheal tube size of $8 \mathrm{~mm}$. A soft NGT-size $14 \mathrm{Fr}$ gauge was attempted by the blind technique. The passage of the tube was smooth, and its gastric placement was confirmed by instilling air into the feeding tube with a syringe while using a stethoscope placed over the stomach to listen for rushing air. A chest X-ray was taken before administering any medication through a feeding tube as per the institute's protocol. The X-ray revealed the course of the NGT into the right lower lobe bronchus [Figure 1]. Air insufflation and epigastric auscultation are commonly used signs of gastric placement. However, good sound transmission may cause misinterpretation even with bronchial misplacement of NGT. Benya et al. reported a $20 \%$ rate of false gastric confirmation by auscultation. ${ }^{[4]}$

The NGT was removed immediately without any complication. Subsequently, a new NGT-size $14 \mathrm{Fr}$ gauge was reinserted by direct laryngoscopy and Magill

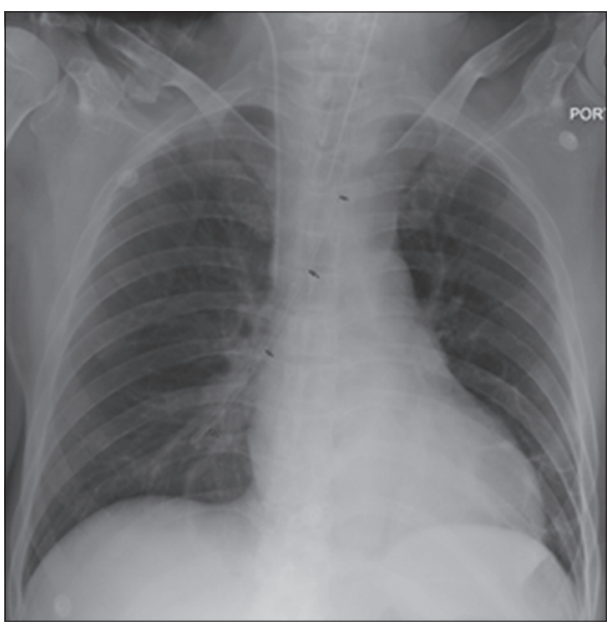

Figure I: Chest X-ray showing nasogastric tube entering right lower lobe bronchus

forceps, but the malpositioning occurred again. This was confirmed by observing bubbles when the end of the feeding tube was placed under water; the appearance of bubbles indicating misplacement of the feeding tube in the respiratory tract.

A senior anesthetist was called for help. He attempted once with the blind technique using a cold (refrigerated) NGT but failed insertion due to coiling in the hypopharynx. Repeat attempt with direct laryngoscopy and Magill forceps was unsuccessful. We then decided to continue the procedure with the aid of video laryngoscope. C-Mac D-Blade video laryngoscope was used, and by visualizing the supraglottic area, the NGT could be easily inserted into the esophageal inlet and anchored at $60 \mathrm{~cm}$. The placement was confirmed by a chest $X$-ray.

Usually, direct laryngoscopy guided by Magill forceps is the technique of choice if NGT insertion is unsuccessful with the blind method. However, the periglottic view seen by direct vision and the narrowing of oral space due to the endotracheal tube are limitations for this approach. In our case, video laryngoscope was very useful for placing the NGT easily and quickly, with less risk of trauma and malposition. Therefore, this method should be considered as an option in difficult situations.

Financial support and sponsorship Nil.

\section{Conflicts of interest}

There are no conflicts of interest. 


\section{T. Kumaravadivel Dharmalingam, Vivekananda Gunasekaran ${ }^{1}$}

Department of Anaesthesiology, Hospital Universiti Sains Malaysia, Universiti Sains Malaysia, 16150 Kubang Kerian, Kelantan, ${ }^{1}$ Department of Anaesthesiology, Faculty of Medicine, Universiti Sultan Zainal Abidin, 20400 Kuala Terengganu, Terengganu, Malaysia

Correspondence: Dr. T. Kumaravadivel Dharmalingam, Department of Anaesthesiology, Hospital Universiti Sains Malaysia, 16150 Kubang Kerian, Kelantan, Malaysia. E-mail: dtkumar123@yahoo.com

\section{References}

1. Tsai YF, Luo CF, Illias A, Lin CC, Yu HP. Nasogastric tube insertion in anesthetized and intubated patients: A new and reliable method. BMC Gastroenterol 2012;12:99.

2. Metheny NA, Meert KL, Clouse RE. Complications related to feeding tube placement. Curr Opin Gastroenterol 2007;23:178-82.

3. Sorokin R, Gottlieb JE. Enhancing patient safety during feeding-tube insertion: A review of more than 2,000 insertions. JPEN J Parenter Enteral Nutr 2006;30:440-5.

4. Benya R, Langer S, Mobarhan S. Flexible nasogastric feeding tube tip malposition immediately after placement. JPEN J Parenter Enteral Nutr 1990;14:108-9.
This is an open access article distributed under the terms of the Creative Commons Attribution-NonCommercial-ShareAlike 3.0 License, which allows others to remix, tweak, and build upon the work non-commercially, as long as the author is credited and the new creations are licensed under the identical terms.

\begin{tabular}{|l|l|}
\hline \multicolumn{2}{|c|}{ Access this article online } \\
\hline Quick Response Code: & Website: \\
& www.ijccm.org \\
\cline { 2 - 2 } & DOI: 10.4103/0972-5229.195756 \\
\hline
\end{tabular}

How to cite this article: Dharmalingam TK, Gunasekaran V. Overcoming a difficult nasogastric tube insertion procedure with a video laryngoscope $\left(\mathrm{C}-\mathrm{Mac}^{\sqrt{ }}\right)$. Indian $\mathrm{J}$ Crit Care Med 2016;20:751-2.

@ 2016 Indian Journal of Critical Care Medicine | Published by Wolters Kluwer - Medknow 